\title{
EL GUSANO DE LAS MUELAS: MEDICINA Y MAGIA SIMPATÉTICA ENTRE LOS MAYAS
}

\author{
Noemí Cruz Cortés \\ Colegio de Historia \\ Facultad de Filosofía y Letras \\ Universidad Nacional Autónoma de México
}

\begin{abstract}
Resumen: Este trabajo se ocupa de una afección física común entre los mayas y en los pueblos mesoamericanos: el dolor causado por un gusano que se come los dientes, llamado "el gusanos de las muelas". La idea para escribir este texto surge de la tradición oral recopilada, por la autora, en la región de los Tuxtlas, Veracruz, donde aún se conserva esta creencia. Al escuchar al narrador, resultó inevitable relacionarlo con las fuentes médicas coloniales y del siglo XIX del área maya que registran este padecimiento y con algunos textos de la zona náhuatl. De esta forma, a través del devenir histórico, podemos analizar a la enfermedad provocada por el gusano de las muelas, comprender los aspectos simbólicos que se involucran en la creación y la curación de esta mal: médicos especialistas, deidades, rezos, plantas, animales y el uso de magia simpatética.
\end{abstract}

Palabras clave: enfermedad, gusano, dientes, simbolismo, magia simpatética.

AвSTRACT: This work deals with a common physical condition in the Mayan and Mesoamerican people: the pain caused by a worm that eats the teeth, called "the teeth worm". The idea to write this text comes from an oral recompilation, by the author, in the Tuxtlas, Veracruz, where this belief still stands. While listening to the narrator, it was inevitable not to relate it with colonial medical sources of the nineteenth century in the Mayan area that recorded this condition, and with some text of the Nahuatl zone. In this form, through historical development, we can analyze the disease caused by the teeth worm, understand the symbolic aspects that are involved in the creation and cure of this disease: specialists, deities, prayers, plants, animals and the use of sympathetic magic.

KeYwords: disease, worm, teeth, symbolism, sympathetic magic.

ReCEPCIÓN: 17 de mayo del 2011.

ACEPTACIÓN: 2 de agosto del 2011. 



\title{
EL GUSANO DE LAS MUELAS: MEDICINA Y MAGIA SIMPATÉTICA ENTRE LOS MAYAS
}

\author{
Noemí Cruz Cortés \\ Colegio de Historia / Facultad de Filosofía y Letras \\ Universidad Nacional Autónoma de México
}

\section{Introducción}

Los temas médicos tienen un lugar preferente en los textos mayas y nahuas, resultan reveladores no sólo acerca de las prácticas terapéuticas, sino que además arrojan luces sobre el concepto de "persona" que tenían estos pueblos, como se ha constatado a lo largo de diversas publicaciones. Este saber ancestral se ha inscrito en los procesos que llama Braudel de "larga duración" (1989: 60), de tal forma que es posible en la actualidad encontrar tradiciones médicas cuyas bases se remontan a tiempos distantes y esquemas conceptuales del mundo prehispánico. Una de estas creencias que ha perdurado tiene que ver con el gusano que se come las muelas, y que aparece en diversas fuentes mayas. Tal vez el pasaje más conocido es el que registra el Popol Vuh; sin embargo, se trata de una creencia difundida también en el área nahua y es probable que se extendiera a toda Mesoamérica.

Aunque este trabajo tiene como punto de partida la tradición oral recopilada en la región de los Tuxtlas, Veracruz, acerca de la curación del dolor de muelas y la extracción del gusano que causa dicho malestar, se trata de un padecimiento que también fue registrado en las fuentes coloniales indígenas y españolas, tanto mayas como nahuas. Con base en dichas fuentes coloniales, se sabe que el dolor de muelas se clasificaba bajo el rubro de enfermedad y afección del cuerpo. El propósito de este estudio es relacionar los datos de las fuentes escritas con la tradición oral de los Tuxtlas, analizarlas y obtener su simbolismo y significación en el devenir histórico de la tradición médica-religiosa mesoamericana. Por lo tanto, iniciaré con el registro de datos, orales y escritos, para después analizar su contenido y su significación con base en los presupuestos metodológicos de la historia de las religiones y su método comparativo.

\section{La historia oral}

El relato de la extracción del gusano de las muelas lo escuché en labios de Casimiro Mena Giles. ${ }^{1}$ Don Casimiro, como lo llaman en su comunidad, es un

\footnotetext{
${ }^{1}$ Agradezco a don Casimiro Mena la gentiliza que tuvo al compartir sus historias y conocimientos
} 
hombre de 88 años de edad, vive desde hace más de 60 años en el ejido Adolfo López Mateos, municipio de Catemaco, Veracruz, enclavado en la sierra de Santa Martha. Es uno de los fundadores del ejido y emigró a los 27 años con su familia desde su pueblo natal, Teloloapan, Guerrero, bajo la promesa gubernamental de tierras para sembrar, a cambio de desmontar la selva veracruzana. ${ }^{2}$ Casado en dos ocasiones, con su primera esposa tuvo 17 hijos y dos con la segunda; aún trabaja la tierra, pues sembrar maíz, frijol y cacahuate es su principal actividad, y de ésta depende la subsistencia de su familia (figura 1).

El ejido López Mateos es un lugar donde convergen distintas tradiciones culturales: la población se integra por veracruzanos, oaxaqueños (sobre todo de la zona de la Mixteca), michoacanos, poblanos, mexiquenses y tabasqueños, entre otros, $\mathrm{y}$, aunque algunos de los ancianos tratan de conservar las costumbres de su tierra natal, a fuerza del paso del tiempo han adoptado tradiciones de esta zona; ${ }^{3}$ sus hijos y nietos nacidos en esta región se identifican como veracruzanos. Un gran porcentaje de la población adulta mayor es analfabeta, o sólo cuentan con los fundamentos básicos de lecto-escritura, así que sus conocimientos e historias son transmitidos de manera oral a las nuevas generaciones; no pocos de estos relatos se inscriben en la tradición oral mesoamericana; por ejemplo: la creencia en un dueño del monte y de los animales y sus ayudantes: los chaneques, las señoras de las fuentes o manantiales, la clasificación de alimentos fríos y calientes (figura 2). No obstante, la extracción del gusano de las muelas es conocida a través de don Casimiro; en la comunidad de López Mateos pocos conservan la creencia del gusano que pudre los dientes, $\mathrm{y}$, aunque siguen utilizando algunos remedios, como masticar tabaco para mitigar el dolor, no realizan ningún tratamiento ni curación.

En el ejido Adolfo López Mateos, la Casa de Salud, como en otras comunidades del país, es visitada por el servicio médico sólo en las campañas de vacunación o de control de epidemias (cólera, paludismos, dengue), el Centro de la Secretaría de Salud más cercano y que cuenta con médico permanente está en Catemaco, a una hora de distancia en automóvil (figura 3). En López Mateos vive don Ángel Abraham, quien conoce de hierbas y remedios tradicionales y su especialidad es

para este trabajo. El primer acercamiento a la comunidad y las charlas se entablan desde el 2005 y terminan en el 2010.

${ }^{2}$ En los años 70, durante el periodo presidencial de Luis Echeverría, se aprobó y puso en marcha el Programa Nacional de Desmontes (Pronade), que dictaminó la incorporación de 25 millones de hectáreas de aptitud forestal a actividades agropecuarias para impulsar la autosuficiencia alimentaria. Las comunidades vegetales mayormente impactadas por los desmontes fueron los bosques tropicales de Veracruz, Campeche, Tabasco y Chiapas.

Por otra parte, la historia de la fundación del ejido es interesante en sí misma, pues se centra en la lucha por las tierras baldías entre los ganaderos y los campesinos; así como en la explotación de maderas preciosas de la región como la caoba.

${ }^{3}$ Esto se refleja en las celebraciones como la del Año Nuevo, donde se quema "el viejo" y se lanzan ilamas, globos de papel de china inflados con aire caliente. La fiesta es amenizada por los jaraneros y el baile en la tarima, que son elementos tradicionales de la región de los Tuxtlas; además de tamales de todos tipos (oaxaqueños, poblanos, etc.) y, en últimas fechas, tiene mucha popularidad el pozole estilo Jalisco o Guerrero. 


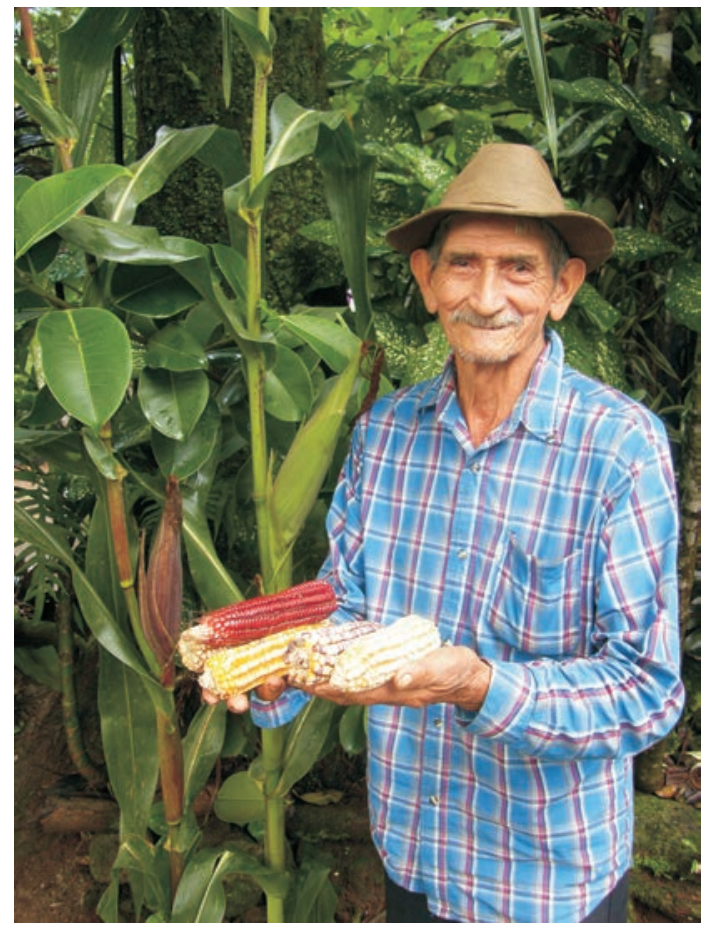

Figura 1. Don Casimiro Mena.

Fotografía de Rubén Cruz, 2011

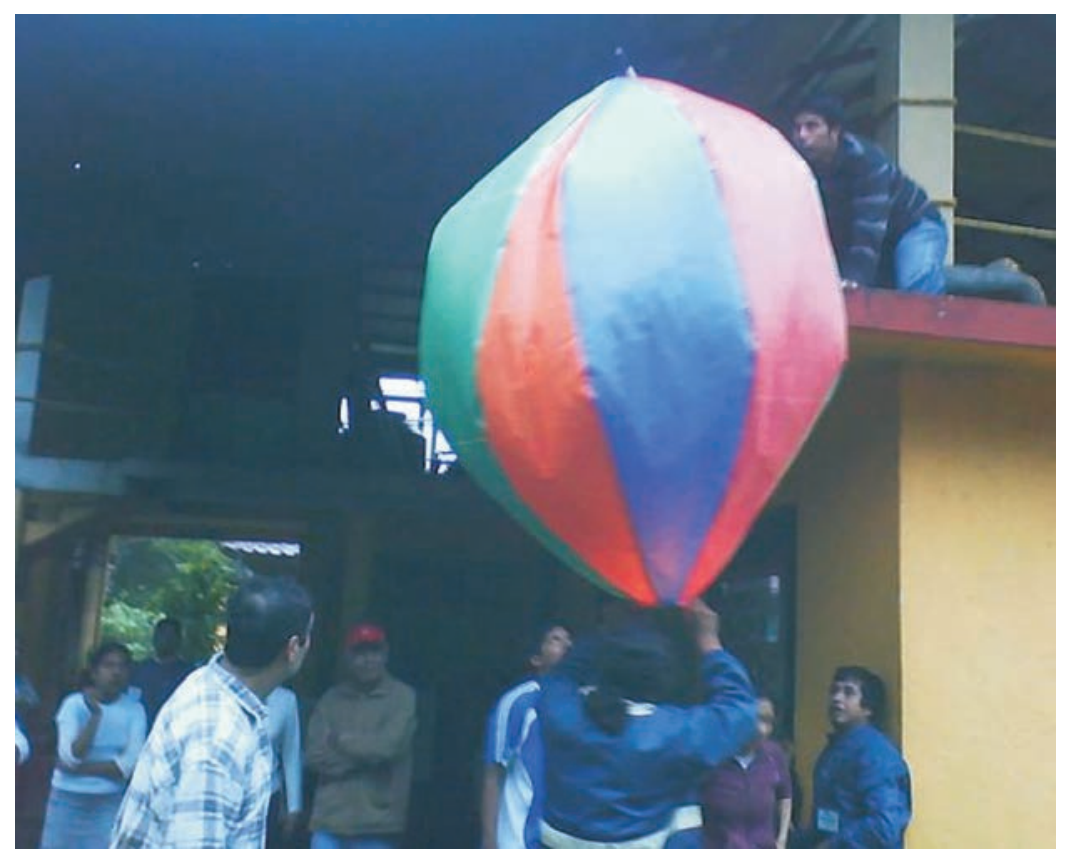

Figura 2. Ilamas, globos de papel de china.

Fotografía de Rubén Cruz, 2009 


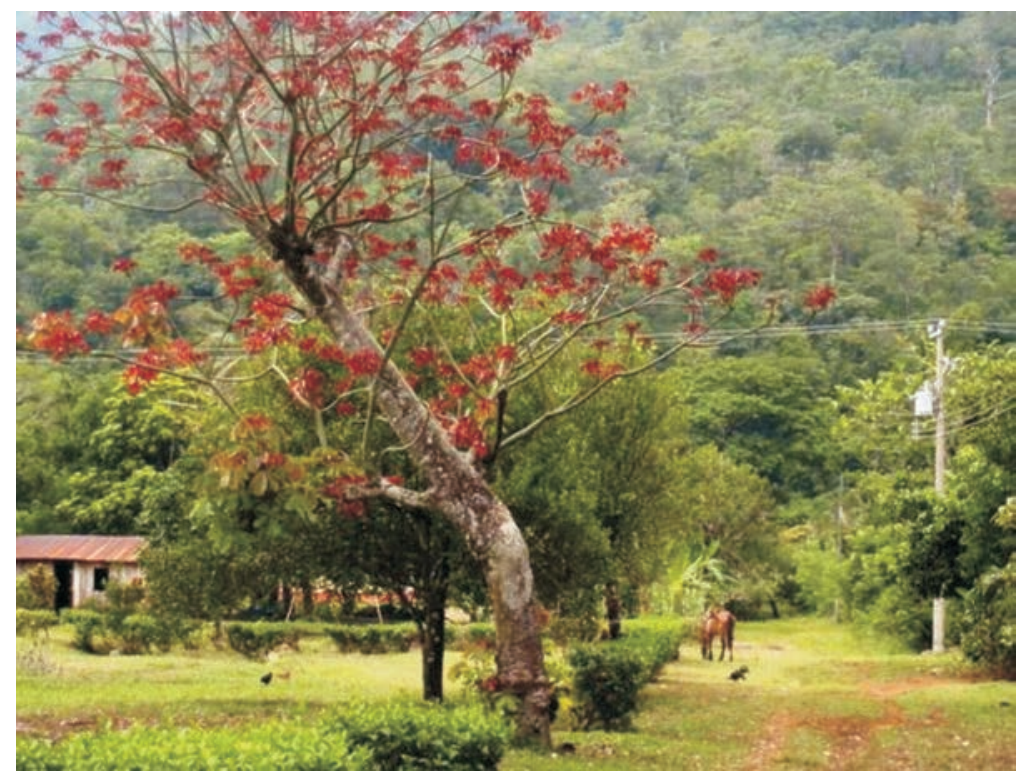

Figura 3. Calle principal del ejido Adolfo López Mateos, Veracruz.

Fotografía de Rubén Cruz, 2009

preparar antídotos contra las picaduras de serpientes, pues abundan en la zona, lo común es que los enfermos recurran a él. Por su parte, don Casimiro no conoce mucho de hierbas, él sabe más de huesos, pero no accede tan fácil a sanar a un enfermo. Generalmente lo hace con sus familiares, con personas cercanas a él o en casos de emergencia, y no pide ningún pago a cambio. Las afecciones de los huesos, entre ellos el dolor de muelas, son padecimientos que él califica como físicos, no menciona en su discurso elementos asociados a algún ser sagrado o a la parte anímica u ontológica del ser humano; ${ }^{4}$ es posible que en este caso el ritual curativo que se involucraba en la restitución de la salud, en la medicina tradicional de los pueblos originarios, se ha ya perdido y sólo queda la parte física de la terapia (figuras 4 y 5).

Don Casimiro hereda la práctica médica fuera de un marco religioso, no recuerda que su abuela o su padre hicieran rezos o algo parecido a un ritual, a pesar de que ambos eran curanderos y sobadores; él sólo aprendió "la técnica" y no tuvo una revelación sagrada que le indicara su profesión médica como le ha

\footnotetext{
${ }^{4}$ Con base en la clasificación que presenta Birman (1996: 19), considero que la afección de los dientes y encías puede corresponder a las enfermedades de origen natural, aquellas que se curaban sin la necesidad de involucrar a las entidades sagradas o algún ritual: "Las enfermedades de origen natural, las más comunes o frecuentes, eran curadas por vías terapéuticas con yerbas y medicamentos; en cambio, las de origen 'divino', las que despertaban temor, derivadas de fuerzas desconocidas y provocadas por maleficios o castigos de los dioses, eran tratadas por especialistas dentro de un terreno mágico religioso a través del ritual".
} 


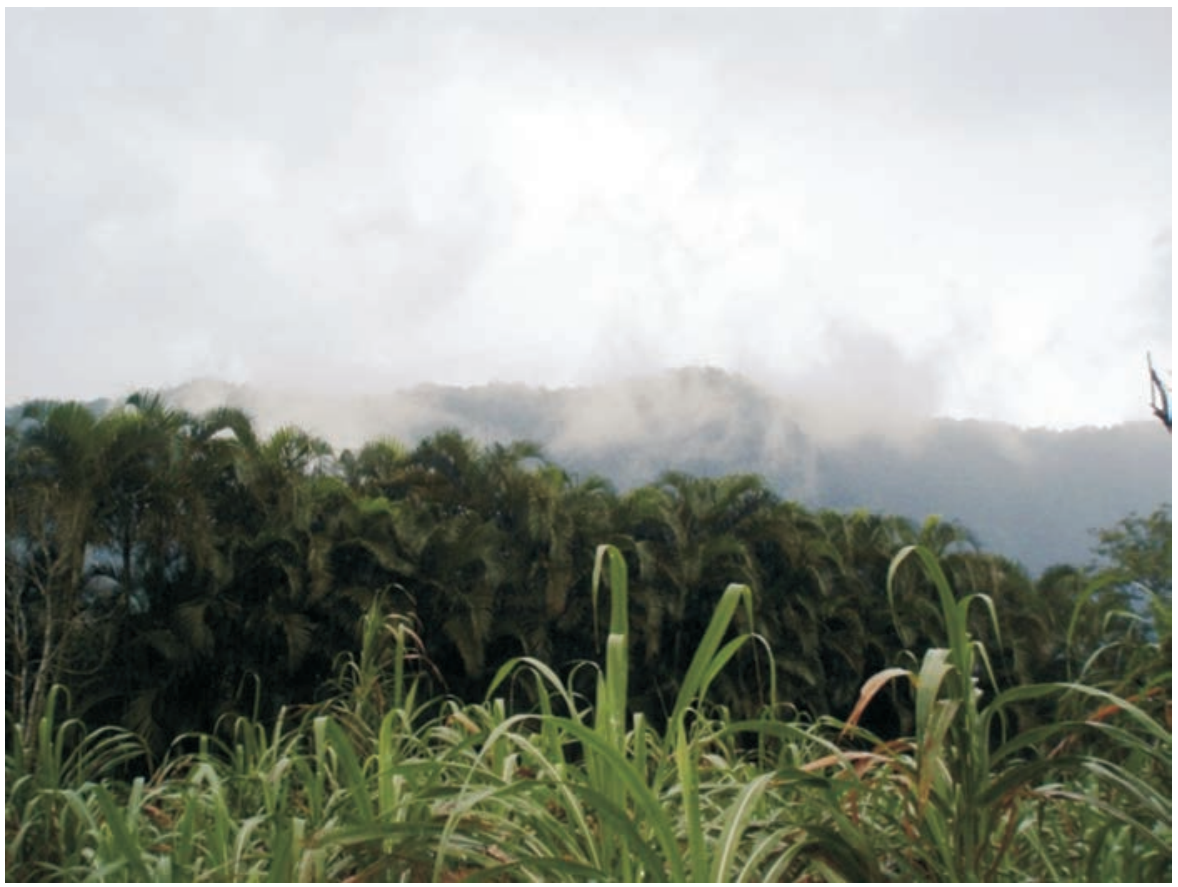

FigurA 4. Milpa, palmar y montaña. Ejido Adolfo López Mateos. Fotografía de Rubén Cruz, 2009

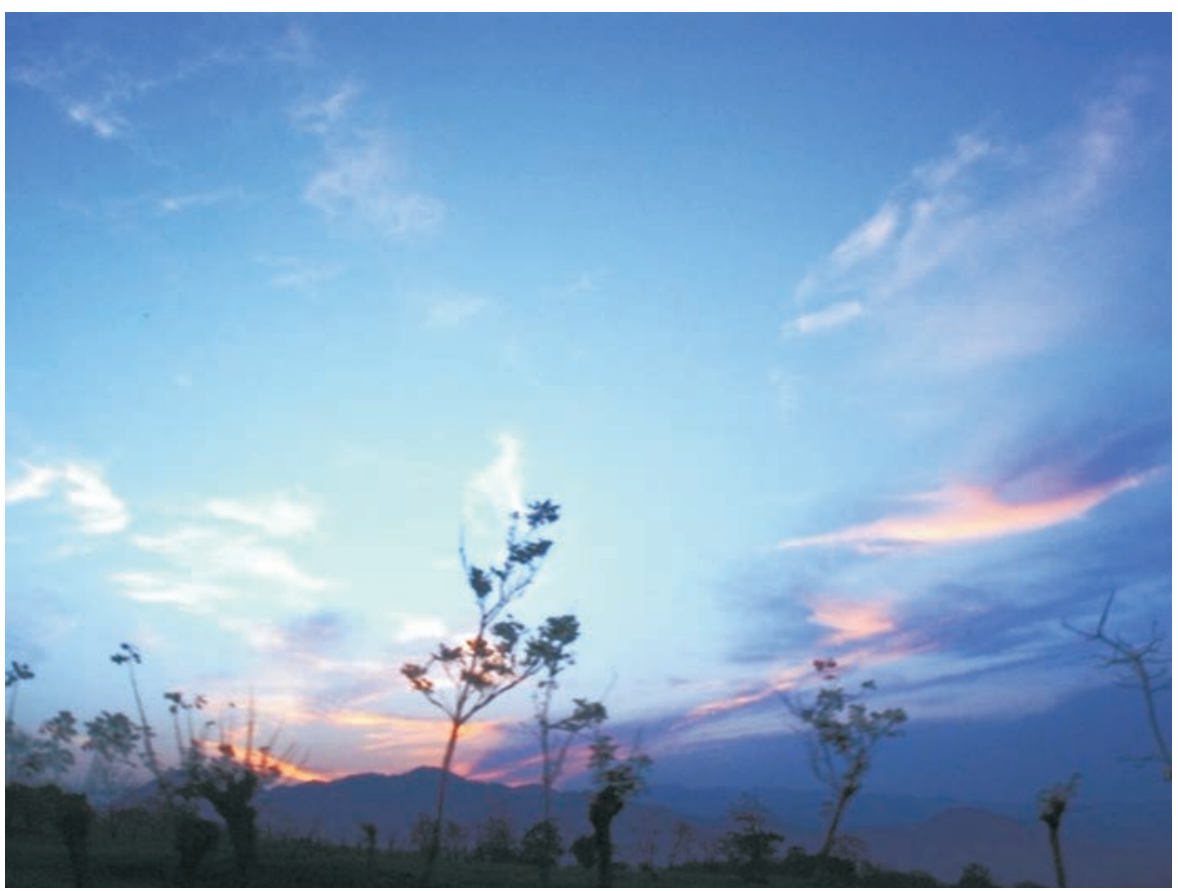

Figura 5. Atardecer en el ejido Adolfo López Mateos.

Fotografía de Rubén Cruz, 2009 
sucedido a varios hombres y mujeres quienes, aunque traten de rechazar el don de curar (lo que le acarrea enfermedades y castigos), terminan por aceptarlo pues es un regalo divino. Por ejemplo, los j-iloletik, o médicos tradicionales tzotziles, comentan que efectivamente son las deidades quienes los eligen y lo saben porque sueñan de manera recurrente con los seres sagrados:

Desde los seis años soñaba todas las noches lo mismo, en mis sueños vi venir de donde sale el Sol dos hombres —un joven y una muchacha-, llegaron desde arriba a llevarme a una casa, al parecer el cielo; me mandó a llamar el Maestro - Jesucristo- que está arriba, y él me dijo que tenía que empezar a curar a todas las personas, tanto hombres, mujeres y niños. "Vas a curar todas las enfermedades", me dijo el Maestro que está en lo alto. Para esto te voy a enseñar todas las medicinas (Gómez, 2005:43).

En este sentido, sin revelación divina y en buena medida sin un contexto religioso parecido al de la cita anterior, don Casimiro rehusó la enseñanza de su abuela y de su padre sin acarrearse ningún problema, sólo conserva la práctica y es, en sentido estricto, un huesero, y como especialista en este ramo su principal terapia son las sobadas. Si bien aprendió de su abuela a "sacar el gusano de las muelas" (puede identificar cuándo es un dolor ocasionado por el gusano y cuándo no lo es, y conoce la enfermedad, su diagnóstico y su cura) porque, pese a su deseo, heredó los conocimientos y la práctica médica tradicional de su familia, en la actualidad no lo practica. ${ }^{5}$

Don Casimiro narra lo siguiente: ${ }^{6}$

Mi abuela era curandera, sabía curar los huesos, sabía otras cosas, pero más sabía de huesos. Me decía: “aprende tú, para que sepas cómo curar a la gente”, pero yo no quería, de todos modos tuve que aprender pues me mandaba por las hierbas y le ayudaba cuando la iban a buscar [los enfermos]. Esto ya tiene tiempo, yo era chiquillo, era allá en Guerrero, en donde nací. También mi padre sabía curar, mi abuela le enseñó. Él también quería que yo aprendiera a curar, pero a mí no me gustaba. Un día mi papá se lastimó el brazo, y me hizo que se lo curara. Me decía: "agárrale aquí, sóbale así, ipero mírame para que veas cómo!”; yo volteaba la cabeza, pero tenía que obedecerlo, y así aprendí a curar los huesos. Mi papá y mi abuela decían: aprende, lo vas a necesitar, en el campo siempre se lastima uno.

\section{En otro momento, don Casimiro platicó lo siguiente: ${ }^{7}$}

\footnotetext{
${ }^{5}$ En realidad no hay una razón específica para que no lo haga. Don Casimiro expresa que "no le gusta”, y en alguna ocasión dijo "para eso ya están los doctores”, refiriéndose a los dentistas.

${ }^{6}$ Comunicación personal, marzo-abril de 2009. Esta cita y las siguientes reproducen el testimonio de Casimiro Mena, son transcripciones y por tanto se respeta su uso del lenguaje. Por otra parte, don Casimiro cree que su abuela era indígena nahua, pero él no se reconoce como nahua, sino como mexicano; es decir, mestizo. No habla ninguna lengua indígena, pues sólo aprendió el español y se declara católico.

${ }^{7}$ En esta cita se indica con las siglas D.C. las frases expresadas por don Casimiro.
} 
D.C. - Mi abuela curaba los dientes, sacaba el gusano de las muelas.

- ¿Y cómo lo hacía?

D.C. —Ella abría la boca [del enfermo], buscaba la muela comida [por el gusano] y ponía hojas de tabaco [verdes], las machacaba, ahí las dejaba un rato hasta que la muela se calentaba. Luego me decía que le calentara cera de Campeche, ${ }^{8}$ y se la ponía en la muela, luego la dejaba un rato. La apretaba y entonces salía el gusano de la muela.

— ¿Usted lo veía? ¿Cómo era?

D.C. - Yo lo veía, era un gusanito, delgado como cabello. Mi abuela decía: "Mira ahí está el gusano, ese es el que se come los dientes".

- ¿Así curaba la muela?

D.C. - No, ya no dolía porque se moría el gusano, y ya no seguía comiendo la muela.

- ¿Y usted ha curado las muelas?, ¿ha sacado el gusano?

D.C. -No, yo no, yo sólo vi, aprendí; eso lo hacía mi abuela. Mira, tú, si quieres tener buenos dientes, para limpiarlos toma un poco de cenizas del fogón y las embarras con tus dedos en tus dientes y luego te enjuagas, así hazlo, y vas a ver: limpiecitos te quedan, como si usaras cepillo. También se limpian con cal.

- ¿De cuál cal?

D.C. - De esa que usan para el nixcomel [nixtamal], una cucharada de cal la echas en un tanto así de agua [como medio vaso], luego haces buches con el agua reposada, ino te comas el asiento!, ini te la tragues [el agua]! Con eso nunca se te pudren los dientes. Hazlo y vas a ver que siempre los vas a tener duros.

Tras este testimonio se observa que don Casimiro conlleva en sus conocimientos parte de la herencia médica de origen mesoamericano. Curar el dolor de muelas, la creencia de un gusano que se come los dientes, el uso de ceniza y cal como abrasivos, la cera o resina y el empleo del tabaco verde como elementos calientes y anestésicos son aspectos que aparecen en los textos médicos recopilados en la época colonial, y que remontan a la época prehispánica, como se verá a continuación.

\section{Los textos mayas coloniales}

Las fuentes mayas registran el mal causado por el gusano de las muelas, sin duda el episodio más conocido se encuentra en el Popol Vuh, cuando Hunahpú e Ixbalanqué le piden a una pareja de ancianos llamados Zaqui-Nim-Ac (el anciano) y Zaqui-Nimá-Tziís (la anciana), que se hagan pasar por sus abuelos y los acompañen ante Vucub-Caquix:

Estos que nos acompañan son nuestros nietos; su padre y su madre ya son muertos; por esa razón ellos van con nosotros, a donde nos dan limosna, pues lo único

${ }^{8}$ Cera natural que produce la melipona, abeja originaria de México y explotada desde tiempos prehispánicos. Entre sus propiedades destaca que es fácilmente moldeable, protectora y aislante. 
que nosotros sabemos hacer es sacar el gusano de las muelas... Mientras tanto, se moría el Señor del dolor de muelas y sólo con gran dificultad podía hablar.

-Yo os ruego encarecidamente que tengáis lástima de mí. ¿Qué podéis hacer? ¿Qué es lo que sabéis curar? les preguntó el Señor. Y los viejos contestaron:

—iOh, Señor, nosotros sólo sacamos el gusano de las muelas, curamos los ojos y ponemos los huesos en su lugar. ... Un gusano es el que os hace sufrir. Bastará con sacar esos dientes y poneros otros en su lugar. ${ }^{9}$

—No está bien que me saquéis los dientes, porque sólo así soy Señor y todo mi ornamento son mis dientes y mis ojos.

- Nosotros os pondremos otros en su lugar, hechos de hueso molido. Pero el hueso molido no eran más que granos de maíz blanco (Popol Vuh, 1990: 36-38).

Los ancianos Zaqui-Nim-Ac y Zaqui-Nimá-Tziís se identifican como sabios, adivinadores, echadores de suertes, son a quienes los dioses creadores consultan sobre la sustancia que formará a los hombres (Popol Vuh, 1990: 28). ${ }^{10}$ Pero es fundamental, para este estudio, destacar su oficio de médicos expertos "en sacar el gusano de las muelas, curar los ojos y poner los huesos en su lugar”.

Por su parte, Margil de Jesús reporta que un hombre confesó haber estado tres días en una cueva como aprendiz de Ixpiyacoc e Ixmucané, quienes le enseñaron "a curar varias enfermedades y en particular de quebraduras de huesos (apud, Garza, 1990: 148). ${ }^{11}$

De acuerdo con Cristina Álvarez (1984: 78) el dolor de muelas no se consideraba una enfermedad propiamente, pues era causado por agentes externos (al igual que las picaduras de víboras, fracturas y torceduras), y por tanto no necesitaba de prácticas rituales pero si de un especialista en huesos como los ancianos del Popol Vuh. Sin embargo, en el Ritual de los Bacabes (1987) aparecen dos conjuros que se utilizaban para extraer el gusano de los dientes, y por tanto implicaría un ritual o al menos la entonación del rezo al momento de curar, y entonces la clasificación de esta afección sería distinta. Un fragmento del primer conjuro dice:

Para los gusanos en las muelas, de los gusanos malignos.

iOh! ¿Será aquí entonces?

¿Serás tú?

Tú, mi Chacal Ik "Viento rojo"

mi Sacal Ik "viento blanco"

mi Ekel $I k$ "viento negro"

mi Kanal Ik "viento amarillo"

\footnotetext{
${ }^{9}$ Edmonson 1971: 40 traduce "just removing jaw bugs", y en la nota 1042 agrega: "FX [Ximénez] and others have translated 'worms' here but the Quiche expression is not that definite; 'Little animals of the jaws' would be fairly literal. The reference may be to a concept of intrusive spirits".

${ }^{10}$ Véase también la nota 3 del preámbulo y la nota 21 de la primera parte del mismo texto.

${ }^{11}$ Según Aguirre Beltrán, para los nahuas entre los ticitl o curanderos, se encontraban el oculista o teixpatiani y el teitzminquí, el huesero (Garza: 1990: 37).
} 
¿Hace ya rato

que le rayaste el corazón

al chulul rojo,

al toncuy rojo,

al tok uil "hambre sangrienta" roja

al ix ek hub rojo (maíz negro)

al ix kan poc (maíz amarillo)...

¿En dónde habrá de tomarse

el corazón del hambre?

¿Qué fue lo que pico?

La aguja roja...

Gusano maligno

tú, el de los ensangrentados huesos

con gusanos

¿Quién fue pues

tu madre?

¿Quién fue tu padre,

el que te dio vida?

Ix Hun Tah Dzib "La de la gran escritura"

Ix Hun Tah Nok "La de los muchos gusanos"...

¿Hace ya rato que escribiste

con la arcilla?

¿Dónde tomaste

el pincel con que escribiste,

es decir, el pincel rojo?

Ahí lo tomaste,

en la orilla de la tierra,

durante el mes chuuen....

el ch'ahum "pájaro carpintero" rojo,

el colonte "pájaro carpintero" rojo.

Aquí se les pescó

muertos de cansancio.

Aquí es donde arrastré

las estrepitosas armas.

Me pongo de pie

para tomarles el fuego.

Los tosté

mientras se asfixiaban,

en los dientes;

del primer hombre de madera

del primer hombre de piedra...

¿Qué árbol le corresponde?

El chacah rojo 


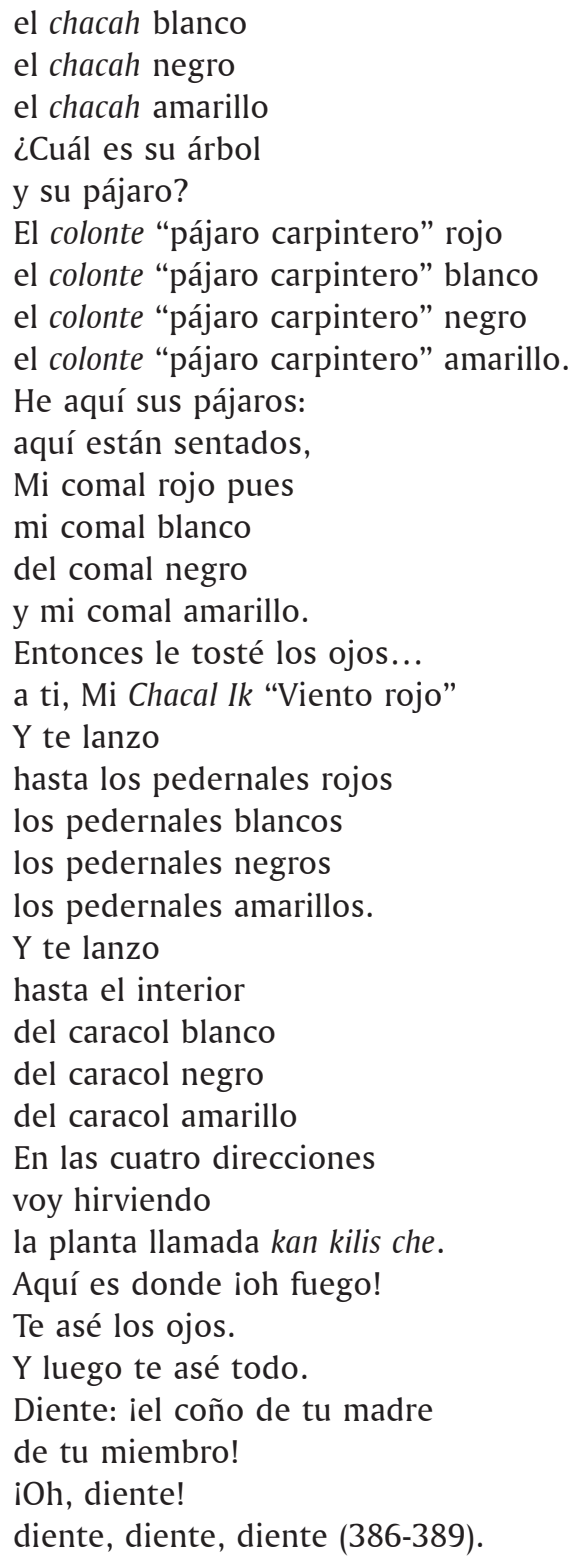

Cabe destacar los elementos fundamentales de este texto de acuerdo al orden en que aparecen: en primer lugar, la comparación del diente con el maíz, el ix ek hub e ix kan poc, que Arzápalo identifica como una variedad del zea mays (nota 152). En segundo lugar señala que la madre de los gusanos es "La de la gran escritura"; es decir, la diosa Ix Chebel Yax de las fuentes coloniales, la diosa 
lunar patrona de la escritura (Cruz, 2002: 55). El pincel y la aguja son elementos iconográficos y simbólicos de esta deidad femenina, y es posible que, a manera de metáfora, la oración equipare pintar la arcilla con picar los dientes. Vientos, árboles, pájaros, comales, pedernales, caracoles son nombrados junto con los cuatro colores del universo, y se asocian con la totalidad de éste. Finalmente, el pájaro carpintero, cuya característica principal es picotear la corteza de los árboles para sacar los insectos que viven bajo de ella; el texto reitera al colonte (kolomte') rojo, color que parece estar ligado al gusano de las muelas, como se verá más adelante. Sin duda, el pájaro carpintero simboliza al médico, ambos realizan la misma labor: extraen los gusanos. Esta significación se refuerza en un fragmento de la segunda oración del Ritual de los Bacabes:

Este texto es para los gusanos también, para aquellos que están en las muelas.

iHun Can Ahau! [El uno serpiente Ahau]. yo seré quien deshaga tu conjuro.

Hace ya rato que nació

el gusano rojizo

el gusano nocturno

el gusano llamado Ix Hun Pedz Kin [La Uno, La mortal]

el gusano de fuego

el gusano despertador

larva hecha gusano.

Yo seré quien deshaga tu conjuro.

Retumbos, retumbos

en el árbol copo;

retumbos, retumbos

en el tzalam;

retumbos, retumbos

en el yaxnic;

retumbos, retumbos

en el kulin che;

retumbos, retumbos

en el kan top'ol can;

retumbos, retumbos

en el chacah.

Con temor

se va yendo

$\mathrm{y}$ es visto

por el ch'ahum "pájaro carpintero" rojo,

el colonte "pájaro carpintero" blanco.

ioh! ¿Será aquí

en donde muerde? 


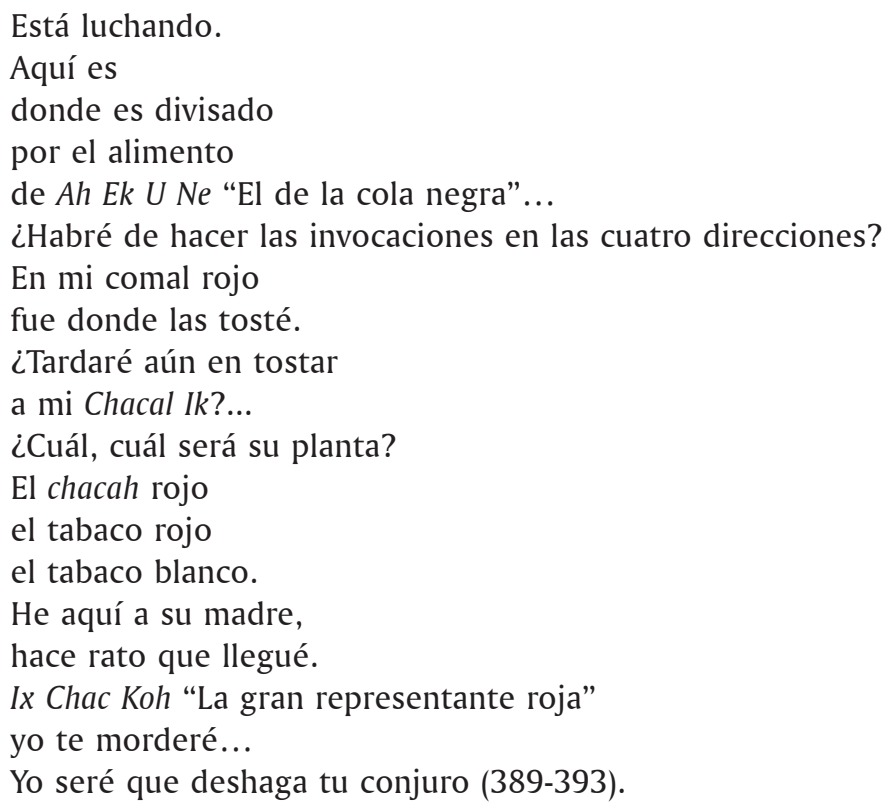

El texto anterior muestra una vez más al pájaro carpintero como el elemento principal utilizado contra los gusanos de las muelas; la reiteración "retumbos, retumbos" relaciona de manera directa el picoteo en el árbol para obligar a los insectos a salir de sus escondites. En el texto maya el vocablo utilizado es booh, booh, que significa "golpear en cosas huecas, o que suena assi" o "golpe de madero hueco" (Calepino Motul, 1995: vol. III: 1518 y Diccionario maya Cordemex, 1980: 60). Además, adquiere un sentido onomatopéyico, porque se asemeja al sonido que emite el ave. El pájaro carpintero es de los pocos animales que se mencionan en este libro médico y también aparece en el Recetario de Indios (1996: 180 ), donde se recomienda su uso como remedio contra el gusano de los dientes y tiene la misma categoría que las plantas curativas. Este texto considera al dolor causado por el gusano de las muelas ( $u$ chibal co $¥$ chaam) como una afección del cuerpo, una enfermedad cuch (cargar, que se carga); si el daño es avanzado, se le da el nombre de kux nokol ti co, laabal, literalmente roer gusanos en el diente, pudrirse $(177,192)$. El procedimiento para curarlo es el siguiente:

[Para el dolor de dientes o de muelas] se toma el pico o la lengua de xkolomte' y se da algunas pinchadas para que sangre la raíz del diente. Si es varón [se le pincha] trece veces, si es mujer nueve veces, hay que darle varias pinchadas; la tzahalche cepillada como fibra y asada junto con la ixcay, se envuelve con algodón y se le pone a la muela, con eso se cura (180).

Al punzar la encía del diente con el pico o la lengua del pájaro carpintero, el médico imita al ave que picotea los árboles para sacar los insectos y larvas en un 
proceso de magia simpatética u homeopática (Frazer, 1956: 35): ${ }^{12}$ aunado a esto, los trece o nueve pinchazos nos vinculan con los niveles celestes y del inframundo, con lo masculino y lo femenino respectivamente; es decir, se involucra en el remedio a símbolos de la cosmovisión maya que adquieren un valor mágico. Pero la función del pájaro carpintero no se limita a los dientes, en los mitos del maíz de los ixiles, chujes y mames de finales del siglo xx; el ave taladra la peña donde las deidades han ocultado el maíz, su picoteo debilita la roca e indica el lugar exacto al rayo para que la rompa y deje al descubierto los granos. En este sentido el ave es una kratofanía del rayo (Cruz, 1999: 104) y ejerce la misma función en los dientes y las muelas, pues los fragiliza y prepara el camino para que el médico extraiga al gusano. De esta manera, con kolomte'-árbol-gusano se establece una relación simbólica que en los rezos y recetas son sustituidos por médico (kolomte')-diente-gusano.

Por otra parte, también se recurre a las hierbas para la curación del dolor de muelas, dice el Recetario de Indios: "Dolor de muelas. La yerba para el dolor de muelas [se toma] el rabo del k'um y del tuk maduro y del op nuevo recién brotado, y el cogollo del chakah y hoja del kate" (1996: 188).

Estas plantas, junto con el tabaco mencionado en el Ritual de los Bacabes, son las más utilizadas por los mayas para extraer el gusano y quitar el dolor de muelas; se trata de anestésicos y desinflamantes ${ }^{13}$ y son complementos terapéuticos después de las punzadas y las sangrías. Todas ellas comparten una naturaleza caliente que se contraponen al gusano de las muelas, "el gusano nocturno" y de naturaleza fría.

No sólo los textos mayas coloniales hablan sobre este malestar, también entre los kekchíes de la primera mitad del siglo xx se conservaba la tradición sobre la curación de los gusanos de las muelas, tal como aparece en un mito recopilado por Thompson en 1930; el fragmento que se cita a continuación trata sobre el enfrentamiento entre K'in, el futuro sol, y el jefe o rey de los zopilotes, con quien X't'actani, la esposa del primero ha huido. Al llegar al territorio del rey zopilote, K'in se aloja en una choza de éste, toma siete granos de maíz rojo, en cada uno hace un hoyo y empieza a tocar la flauta y el tambor, produciéndole un gran dolor de muelas a su anfitrión:

\footnotetext{
${ }^{12}$ Frazer explica que la magia simpatética "establece que las cosas se actúan recíprocamente a distancia mediante una atracción secreta, una simpatía oculta, cuyo impulso es transmitido de la una a la otra por intermedio de lo que podemos concebir como una clase de poder invisible" (1956: 35).

${ }^{13}$ Para la identificación de algunas de las plantas mencionadas, recomiendo consultar el apéndice del Recetario de indios; por ejemplo, para op, k'um y tuk, véase la página 260, 257 y 263, respectivamente. En estos tres ejemplos se observa que son plantas y frutos utilizados también para quemaduras y piquetes de animales ponzoñosos para mitigar el dolor y la hinchazón. Véase también el Calepino de Motul (volumen III: 1644) donde se dice que el chacah es un árbol que se utiliza para la hinchazón, las llagas y el salpullido.
} 
El jefe (zopilote) comenzó a sufrir de dolor de muelas (causado por los granos de maíz con hoyos). Gradualmente el dolor empeoró. El jefe envío a su sirviente a preguntar a Lord K'in si él podría curar el dolor de muelas (Thompson, 1930: 131). ${ }^{14}$

K'in acepta curar al jefe de los zopilotes, pero éste no le permite ingresar a su casa pues ahí estaba X't'actani. Entonces K’in continúa tocando la flauta y el tambor y con ello aumenta el dolor del zopilote; sólo se detiene hasta que éste lo deja entrar:

Cuando [K'in] entró en la habitación, él vio a su esposa sentada allí con el jefe enfermo. Por medio de su destreza rápidamente curó al hombre enfermo de su dolor de muelas y mientras el jefe caía dormido, Lord K'in se volvió a X't'actani y la obligo a huir con él (Thompson, 1930: 131). ${ }^{15}$

Una vez más, el dolor de muelas y su curación se utiliza como artimaña para acercase al enemigo y derrotarlo; en el Popol Vuh, Vucub Caquix es despojado con la misma estrategia de sus atuendos de riqueza y belleza, como se señaló anteriormente.

En otro aspecto, además de ser una deidad solar, la cita anterior nos revela que K'in también es experto en provocar y remediar el dolor de las muelas a través del uso de magia simpatética: el maíz representa a las piezas dentales y los hoyos simbolizan las perforaciones causadas por los gusanos. De hecho, la voz maya co se usa de manera indistinta para diente y grano de maíz (Swadesh, Álvarez y Bastarrachea, 1991: 40), y según Álvarez (ibid.: 338) la palabra xoh (carcomido) se aplica tanto a los dientes como al maíz carcomido, agujereado y medio podrido. Esto permite encontrar otra sustitución no sólo física, sino también simbólica: el maíz por el diente.

Por otra parte, en los textos consultados resalta la relación entre el color rojo y el padecimiento de los dientes: el pájaro carpintero rojo, los vientos rojos, los gusanos rojos, el chacah rojo, el tabaco rojo y la deidad Ix Chac Koh "La gran representante roja" (que tal vez se relaciona con la anciana diosa roja, la diosa $\mathrm{O}$ de los códices) y los granos de maíz rojos. Si bien es cierto que para los mayas este color está asociado al oriente, al nacimiento del sol, la relación con el gusano de las muelas no está del todo clara; es posible que aluda a los elementos de naturaleza caliente que se necesitan para sanar a la afección.

${ }^{14}$ La traducción de éste y el siguiente fragmento es mía: "The chief began to suffer from toothache (caused by the red corn with the holes). Gradually the pain grew worse. The chief sent his servant to ask Lord Kin if he could cure the pain" (Thompson, 1930: 131). Como se aprecia en esta cita, Thompson llama a la deidad solar "Lord K'in", este nombre lo utiliza en otras publicaciones cuando hace referencia a este mito (tal parece que le otorga un título nobiliario) y por tanto decidí respetar la redacción del autor.

15 "When he entered the room, he saw his wife sitting there with the sick chief. By means of his skill he quickly cured the sick man of his toothache, and in a short while the chief fell asleep. Lord Kin turned to X't'actani and bade her flee with him". 
En este sentido, chac se traduce como "cosa colorada", pero se trata de una palabra polisémica; por ejemplo: chac "cocer en agua o en otro licor"; chac "en composición, muy o mucho"; chac "agua, en algunas maneras de decir: ya entran o comienzan las aguas"; chacal haa "aguazero grande con truenos y relámpagos que dura que por algunos días"; kaxal u cah chac "llueve con truenos" (Calepino Motul, 1995: vol. III: 1644); chac "agua o aguacero que llueve"; u cum chac "las borbotijas a manera de cascabeles que haze el agua quando llueue" (Bocabulario de Maya Than, 1993: 72). Con base en lo anterior, chac adquiere otro valor simbólico, pues no sólo es rojo o caliente, también es abundancia, agua en exceso, elementos que generan fertilidad, pero también putrefacción; de esta manera es viable suponer que chac funciona en dos sentidos: rojo-caliente-seco-cocido y lluvia-abundancia-húmedo-podrido, y ambos se relacionan con el gusano de las muelas y su curación.

\section{Los textos nahuas coloniales}

En las fuentes nahuas analizadas ${ }^{16}$ no se habla del gusano de las muelas ni de su extracción, pero sí del dolor de los dientes causado por la caries, cuyo remedio principal es el tabaco o pícietl. Sahagún, en los "Textos de Tepepulco", clasifica a este malestar como una enfermedad del cuerpo humano: "se curan con pícietl y raíz de pópotl, que aquí se dan" (López, 2000: 49) y, en los "Textos de MéxicoTlatelolco", la receta que recopila incluye polvo de gusano y sangrías:

Caries. Su curación es mezclar ocótzotl [polvo del gusano llamado] conyayáhual. Esto se pone con pluma menuda por encima. Y por dentro se presiona el diente con chile caliente, y se presiona el diente con sal, y se punzan mucho nuestras encías, y se pone en nuestros dientes tlalcacáhuatl. Si nada reduce [el mal], se hace sacar nuestro diente. Ahí [en el hueco] se pone sal (Sahagún, "Textos de México-Tlatelolco", ibid: 53).

Entre diversos grupos indígenas contemporáneos existe la creencia de que algunos "brujos", en especial hombres ancianos, pueden desprenderse de su carne hasta quedarse sólo con su esqueleto y así elevarse por los cielos. Para destruirlo se debe echar a la carne del brujo sal y chile seco, tostado y molido para cocerla, y así no pueda pegarse a los huesos de su dueño y entonces ocasionar su muerte. ${ }^{17}$ Por lo anterior, resulta interesante que este texto nahua hable del uso del

\footnotetext{
${ }^{16}$ Los textos consultados se encuentran recopilados en López Austin, Alfredo, Textos de medicina náhuatl, 2000.

${ }^{17}$ Véase, por ejemplo, "El brujo que se convirtió en esqueleto”, cuento tzotzil; "El esqueleto volador", cuento tojolabal; ambos publicados en diferentes números de la serie Cuentos y relatos indígenas del Centro de Investigaciones Humanísticas de Mesoamérica y el Estado de Chiapas, Universidad Nacional Autónoma de México.
} 
chile y sal para este padecimiento, ambos elementos calientan al diente; es decir, secan o cuecen aquello que ocasiona el dolor y también a la carne descubierta tras la extracción de la pieza dental.

Por su parte, el códice Libellus de Medicinalibus Indorum Herbis, de Martín de la Cruz, contiene una receta para el dolor de dientes:

Los dientes enfermos y cariados deberán punzarse con un diente de cadáver. En seguida se muele y se queman la raíz de un alto arbusto llamado teonochtli, juntamente con un cuerno de venado y estas piedras finas: íztac quetzaliztli y chichíltic tapachtli, con un poco de harina martajada con algo de sal. Todo esto se pone a calentar. Toda esta mezcla se envuelve en un lienzo y se aplica por breve tiempo apretada con los dientes, en especial con los que duelen o están careados. En último lugar se hace una mezcla de incienso blanco y una clase de untura que llamamos xochiocótzotl y se quema a las brasas y su olor se recoge en una mota gruesa de algodón que se aplica a la boca con alguna frecuencia o mejor se ata a la mejilla (88).

Esta cita muestra un procedimiento similar a los anteriores: punzadas y la aplicación local de elementos calientes; no obstante, es más complejo pues involucra un diente de cadáver como instrumento médico, cuerno de venado y piedras; destaca que el olor de la mezcla se capture en un algodón, lo que indica que la sustancia ligera, la esencia que viaja en el humo, ayuda a mejorar el malestar causado por los dientes careados. Así esta receta abarca elementos de alto valor simbólico que sólo podía manejar un experto en estos menesteres como el tetlacuicuilique o tetlacuicuiliani, chupador, un curandero que se especializaba en sacar gusanillos de la boca y los ojos, pedrezuelas de otras partes del cuerpo (Garza, 1990: 37).

Sobre los consejos para cuidar los dientes, los textos nahuas coloniales incluyen recetas similares a las referidas por don Casimiro Mena; por ejemplo, Sahagún, en los "Textos de México-Tlatelolco", escribe que, para no contraer la denominada "fiebre acuática de los dientes", los alimentos no deben ingerirse muy calientes, sino tibios, o bien esperar a que los dientes se enfríen para beber o comer alimentos fríos (Garza, 1990: 53); don Casimiro dice que "los dientes se estrellan como el vidrio con lo caliente y luego lo frío".

Estas fuentes también hablan de la limpieza de la dentadura: "si se queda atorada la carne en los dientes de la gente, los quema, los acaba, pudre nuestros dientes" (Garza, 1990), por eso nos dice el texto:

Se raspará con carbón. Se limpiarán, se harán lucir nuestros dientes con sal, se lavarán los dientes con tlatlauhcapatli lleno de sal, lleno de chile, y un poco de [esta] medicina remojada la pondrá, la coloca en sus dientes. Con esta medicina raspará los dientes, se lavará la boca... Y se lavarán nuestros dientes muchas veces con [agua de] cuauhtepuztli o se pondrá [polvo de su corteza] en nuestros dientes, y se raspará el sarro (53-54). 
El carbón, polvo de corteza o cenizas, sal y chile funcionan como abrasivos para limpiar los dientes; los dos primeros elementos también son mencionados por don Casimiro para el cuidado de la dentadura y, en la región mazahua del estado de México, los ancianos continúan utilizando la tortilla quemada o carbonizada, para tallarse los dientes después de comer. ${ }^{18}$ Lo anterior demuestra que los conocimientos de don Casimiro se inscriben en la tradición médica mesoamericana.

\section{Conclusiones}

El relato de Casimiro Mena sobre la extracción del gusano de las muelas muestra la pervivencia de algunas creencias médicas de origen mesoamericano, las cuales fueron registradas en las fuentes coloniales mayas y nahuas, y permanecen en su estructura, a lo largo del devenir histórico y a través de la oralidad hasta hoy en día. El testimonio de don Casimiro incluye elementos fundamentales que aparecen también en los textos indígenas coloniales: el tabaco, las cenizas y el uso de los conceptos frío y caliente, todos estos afines a la cosmovisión mesoamericana. Don Casimiro es partícipe de estas nociones desde pequeño a través de su abuela y su padre, especialistas en curar huesos, en su poblado de origen, Teloloapan, lugar de filiación náhuatl. A pesar de pertenecer a una familia de médicos tradicionales, la negación de don Casimiro para aprender la profesión no significó ningún riesgo en su integridad en tanto que persona: es heredero de los conocimientos, pero no del plano simbólico que acompaña a muchos curanderos tradicionales. Las experiencias adquiridas en Teloloapan las llevó consigo al Ejido López Mateos en los Tuxtlas, Veracruz, donde las comparte a través de sus relatos, o bien, las pone en práctica cuando es requerido para componer o sobar los huesos de un vecino o familiar.

Por otra parte, el malestar que generan los gusanos comedores de muelas y dientes, que a su paso dejan hoyos y ocasionan el dolor, se inscribe, como ya se ha mencionado, en los padecimientos y afecciones físicas causadas por factores externos; $y$, si bien no era necesario realizar rezos o rituales para atacar este tipo de males (entre los nahuas, bastaba con capturar en un algodón el olor que desprendían las plantas y el incienso en las brasas y colocarlo en la encía del diente enfermo), para los mayas debía tener suma importancia y era indispensable, en ocasiones, conjurar a las deidades para eliminarlo, como lo demuestra el Ritual de los Bacabes.

Los elementos involucrados en los conjuros mayas y en las recetas nahuas poseen una alta significación simbólica: el tabaco, elemento de naturaleza caliente, anestésico efectivo contra el dolor de huesos, pero sobre todo la planta predilecta de los chamanes en los rituales curativos, y asociado con las deidades de

${ }^{18}$ Carlos Cruz, mazahua. Comunicación personal, Jocotitlán, estado de México, agosto del 2009. 
orden solar. El pájaro carpintero es utilizado para liberar la presión de la encía, pero más allá de su uso terapéutico se reviste de un alto valor simbólico: es equiparado al médico pues ambos buscan y sacan los gusanos. Su pico y su lengua se convierten en instrumentos kratofónicos en el momento de la curación, ya que concentran el poder, la fuerza y la destreza de esta ave, que, si bien no es representada plásticamente por los mayas, su presencia en los textos coloniales y en los mitos del siglo xx nos hablan de un ave sagrada, vinculada con los hombres como proveedora de salud y del alimento.

Por otra parte, el picotear del pájaro carpintero en el árbol, los pinchazos en las encías (trece o nueve veces dependiendo del sexo del paciente), la sustitución de los dientes por granos de maíz, las perforaciones realizadas en los granos rojos, la música para producir dolor muestran el uso de la magia denominada simpatética donde la acción sobre un objeto repercute en un individuo.

En otro aspecto, el saber curar el dolor de muelas y exterminar a los gusanos juega un papel importante en las narraciones míticas: se utiliza para engañar y derrotar al enemigo; poseer los conocimientos necesarios para sanar los huesos y las muelas permite a la pareja de ancianos del Popol Vuh acercarse a Vucb Caquix y despojarlo de aquello que lo reviste de honra: sus dientes y muelas; y, en el mito kekchí, el saber curar el malestar hace posible que Kin recupere a su esposa. Considero que sustituir los dientes y las muelas por granos de maíz obedece a cuestiones no sólo físicas, sino también simbólicos, así lo demuestran los conjuros del Ritual de los Bacabes.

Pero esta enfermedad debe ser tratada por los especialistas, Zaqui-Nim-Ac y Zaqui-Nimá-Tziís son expertos en huesos, dientes y ojos; es decir, médicos de afecciones externas, y Kin no sólo genera el malestar, también la cura. Asimismo, los seres sagrados relacionados al dolor de dientes son Ix Chac Koh, la anciana roja, la gran anciana; Ix Hun Tah Dzib, la de la gran escritura; e Ix Hun Tah Nok, la de los muchos gusanos, llamadas cada una "la madre" de los gusanos. Se trata de nombres o títulos femeninos de una deidad que es posible identificarla con la diosa $\mathrm{O}$ de los códices mayas; esta hipótesis se refuerza porque es una diosa anciana, de aspecto terrestre, asociada a la noche, a lo frío, lo femenino, a lo crudo, a los "los gusanos nocturnos" que generan lo podrido.

Los gusanos de las muelas también surgen del exceso de calor emanado por los residuos de la comida y de la carne atorada en las piezas dentales; no obstante, la naturaleza de estos animales es sin duda fría, conciben lo descompuesto, y deben contrarrestarse con elementos calientes para restituir la salud: tabaco, chile, sal, cenizas, cal y cera eliminan lo putrefacto, matan al gusano y limpian los dientes.

Sin embargo, aún hay un elemento cuyo significado no es del todo claro: el color rojo, constante en las fuentes mayas: pájaro carpintero, gusanos, granos de maíz, tabaco, árboles, diosas, agujas, vientos. En primera instancia se asocia al oriente, sobre todo cuando en los conjuros del Ritual de los Bacabes se mencionan los cuatro rumbos del universo; o bien, este color podría ir en función del calor 
y del fuego por su asociación solar. Sin embargo, con base en el sentido polisémico de la palabra chac, es posible que se relacione con el exceso de humedad ocasionada por la lluvia y que genera putrefacción y con ella la aparición de los gusanos. Así, no sólo es el color rojo lo que ayuda a comprender valor simbólico de la voz chac, sino la dicotomía que ofrece: caliente-seco-cocido y frío-húmedopodrido. Lo primero, de carácter benigno; y el segundo, dañino.

Así, tras la revisión en la tradición escrita y oral, se confirma la pervivencia de una serie de nociones relacionadas a la limpieza y conservación de la dentadura, pero sobre todo destacan las creencias en torno a los gusanos devoradores de las muelas. El testimonio de don Casimiro Mena es una muestra de que estos aspectos de la medicina de origen mesoamericano continúan presentes en la actualidad, y que no se circunscriben a un espacio determinado; pues, aunque don Casimiro sea originario del estado de Guerrero, su relato, que gentilmente ha compartido, pertenece ahora también a la región de los Tuxtlas. La oralidad juega un papel determinante en las tradiciones de los pueblos originarios, pues conserva los nódulos de su cosmovisión a lo largo del tiempo, hace que permanezcan, que perduren, al igual que los huesos y los dientes.

\section{BIBLIOGRAFÍA}

Álvarez, Cristina

1984 Diccionario etnolingüístico del idioma maya yucateco colonial. Vol. II: Aprovechamiento de los recursos naturales. México: Universidad Nacional Autónoma de México, Instituto de Investigaciones Filológicas, Centro de Estudios Mayas.

Birman Furman, Raquel

1996 "Estudio introductorio", Recetario de indios en lengua maya. Índices de plantas medicinales y de enfermedades coordinados por D. Juan Pío Pérez con estratos de los recetarios, notas y añadiduras por C. Hermann Berendt, M.D., Mérida 1870. México: Universidad Nacional Autónoma de México, Instituto de Investigaciones Filológicas, Centro de Estudios Mayas, 13-60.

Bocabulario de Maya Than. Codex Vindobonensis N.S. 3833

1993 René Acuña (ed.). México: Universidad Nacional Autónoma de México, Instituto de Investigaciones Filológicas, Centro de Estudios Mayas.

Braudel, Fernand

1989 La Historia y las ciencias sociales. México: Alianza Editorial (Sección: Humanidades).

Calepino de Motul. Diccionario maya-español

1995 Vol. 3, Ramón Arzápalo (ed.). México: Universidad Nacional Autónoma de México, Instituto de Investigaciones Antropológicas. 
Cruz Cortés, Noemí

1999 "Mitos de origen del maíz de los mayas contemporáneos", tesis de maestría en Estudios Mesoamerianos. México: Universidad Nacional Autónoma de México, Facultad de Filosofía y Letras/Instituto de Investigaciones Filológicas.

2002 "Las señoras del pincel”, Nueva Gaceta Bibliográfica, número especial. 5 (20): 54-60, octubre/diciembre. México: Universidad Nacional Autónoma de México, Instituto de Investigaciones Bibliográficas.

Diccionario maya Cordemex

1980 Alfredo Barrera Vázquez, et al. (eds.). Mérida: Cordemex.

Frazer, James

1956 La rama dorada. México: Fondo de Cultura Económica.

Garza, Mercedes de la

1990 Sueño y alucinación en el mundo náhuatl y maya. México: Universidad Nacional Autónoma de México, Instituto de Investigaciones Filológicas, Centro de Estudios Mayas.

Gómez Sántiz, María Magdalena

2005 Jiloletik, médicos tradicionales de los Altos de Chiapas. Tuxtla Gutiérrez: Gobierno del Estado Chiapas.

López Austin, Alfredo

2000 Textos de medicina náhuatl, 5a ed. México: Universidad Nacional Autónoma de México, Instituto de Investigaciones Históricas (Monografías, 19).

Popol Vuh. Las antiguas historias del Quiché.

199021 reimp. México: Fondo de Cultura Económica.

Recetario de indios en lengua maya. Índices de plantas medicinales y de enfermedades coordinados por D. Juan Pío Pérez con estratos de los recetarios, notas y añadiduras por C. Hermann Berendt, M.D., Mérida 1870.

1996 Raquel Birman Furman (ed.). México: Universidad Nacional Autónoma de México, Instituto de Investigaciones Filológicas, Centro de Estudios Mayas.

Ritual de los Bacabes

1987 Ramón Arzápalo (ed.). México: Universidad Nacional Autónoma de México, Instituto de Investigaciones Filológicas, Centro de Estudios Mayas.

Swadesh, Mauricio, María Cristina Álvarez y Juan Ramón Bastarrachea

1991 Diccionario de elementos del maya yucateco colonial. México: Universidad Nacional Autónoma de México, Instituto de Investigaciones Filológicas, Centro de Estudios Mayas (Cuaderno, 3). 
The Book of Counsel: The Popol Vuh of the Quiche Maya of Guatemala

1971 Munro S. Edmonson (ed. y trad.). Nueva Orleans: Tulane University (MARI publication, 35).

Thompson, J. Eric

1930 Ethonolgy of the mayas Southern and Central British Honduras. Chicago: Field Museum of Natural History. 
